EPJ manuscript No.

(will be inserted by the editor)

\title{
Improving the convergence of the chiral expansion for nuclear forces II: low phases and the deuteron
}

\author{
E. Epelbaum ${ }^{1}$ a, W. Glöckle ${ }^{1}$ b , and Ulf-G. Meißner ${ }^{2}$ c \\ 1 Ruhr-Universität Bochum, Institut für Theoretische Physik II, D-44870 Bochum, Germany \\ ${ }^{2}$ Universität Bonn, Helmholtz-Institut für Strahlen- und Kernphysik (Theorie), Nußallee 14-16, D-53115 Bonn \\ Received: date / Revised version: date
}

\begin{abstract}
Recently we have proposed a new cut-off scheme for pion loop integrals in the two-pion exchange potential. This method allows for a consistent implementation of constraints from pion-nucleon scattering and has been successfully applied to peripheral nucleon-nucleon partial waves. We now consider low partial waves in the non-perturbative regime, where the regularized Lippmann-Schwinger equation has to be solved in order to generate the bound and scattering states. We observe an improved description of most of the phase shifts when going from next-to- to next-to-next-to-leading order in the chiral expansion. We also find a good description of the deuteron properties. In addition, the new cut-off scheme allows to avoid the presence of unphysical deeply bound states. We discuss the cut-off dependence of the four-nucleon lowenergy constants and show that their numerical values can be understood in terms of resonance saturation. This connects the effective field theory approach to boson exchange phenomenology.
\end{abstract}

PACS. 13.75.Cs Nucleon-nucleon interactions - 21.30.-x Nuclear forces - 12.39.Fe Chiral Lagrangians

\section{Introduction}

In Ref. [1] (which is called I form here on), we have presented a method to improve the convergence of the chiral ' expansion for the nucleon-nucleon (NN) interaction based on spectral function regularization. In earlier approaches, an unphysically strong attraction in the isoscalar central part of the chiral two-pion-exchange (TPE) at next-tonext-to-leading (NNLO) order in the chiral expansion was found. This is due to the high-momentum components of the exchanged pions, which appear when using dimensional regularization (or equivalent schemes), and which cannot be properly treated in the corresponding effective field theory (EFT). Using a cut-off (or spectral function) regularization instead of the dimensional one and taking reasonable values for the momentum space cut-off allows to remove spurious short-distance physics associated with high-momentum intermediate states and to greatly improve the convergence of the chiral expansion. In particular, one can use without problems the values of the dimension two low-energy constants (LECs) $c_{i}$ consistent with elastic pion-nucleon scattering data. More precisely, in I we have considered the spectral functions obtained from the next-to-leading order (NLO) and NNLO TPE contributions and argued that only masses below the chiral symmetry breaking scale should be taken into account

\footnotetext{
${ }^{a}$ email: evgeni.epelbaum@tp2.ruhr-uni-bochum.de

b email: walter.gloeckle@tp2.ruhr-uni-bochum.de

c email: meissner@itkp.uni-bonn.de
}

explicitly in the loop integrals while shorter range contributions have to be represented by contact interactions. This can be easily implemented by applying a cut-off to the spectral functions. We have also proposed a simple and convenient way to derive analytic expressions for regularized TPE in the momentum space based on the spectral function representation.

In I, we have considered the peripheral partial waves $(l \geq 2)$, because at NNLO, these are given entirely by onepion-exchange (OPE) and TPE with no free parameters. We have calculated these phases in Born approximation which should be legitimate at least for the $\mathrm{D}$ - and higher waves. The results for the $\mathrm{D}$ - and $\mathrm{F}$-waves are still not completely converged at NNLO, but the error of a few $(1)^{\circ}$ at $E_{\text {lab }}=300 \mathrm{MeV}$ for the $\mathrm{D}-(\mathrm{F}-)$ waves appears reasonable. There is no breakdown of the chiral expansion for $\mathrm{D}$-waves beyond $T_{\mathrm{lab}}=50 \mathrm{MeV}$ and for $\mathrm{F}$-waves beyond $T_{\text {lab }}=150 \mathrm{MeV}$ as found earlier using dimensional regularization. In this paper, we apply our method (cutoff regularization, CR, for short) to the low partial waves in the non-perturbative regime, where we have to solve the regularized Lippmann-Schwinger equation to generate the bound and scattering states. As will be demonstrated, there are no deeply bound states (for a reasonable range of cut-offs in the spectral function representation of the effective potential and regularized Lippmann-Schwinger equation), and low-energy observables are not affected by CR. 
Our manuscript is organized as follows. In section 2 we briefly review the formalism detailed in I and discuss the regularization of the Lippmann-Schwinger equation. In section 3 we apply the formalism to $n p$ partial waves, with particular emphasis on the low phases ( $\mathrm{S}_{-}$and $\mathrm{P}-$ waves). We also discuss the range parameters in the $\mathrm{S}_{-}$ waves and the deuteron properties and compare to the results obtained in dimensional regularization (DR). The physics behind the values of the low-energy constants accompanying the four-nucleon operators is also elucidated. The summary and conclusions are given in section 4

\section{Formalism}

In I we have calculated the NN potential at NNLO in the low-momentum expansion using cut-off regularization in the spectral function representation. For completeness, we briefly review the pertinent formalism here.

The chiral TPE potential up to NNLO can be decomposed into isoscalar and isovector central, spin-spin and tensor components, generically called $W_{i}(q)$ here. These functions $W_{i}(q)$ can be represented by a continuous superposition of Yukawa functions (modulo subtractions)

$$
W_{i}(q)=\frac{2}{\pi} \int_{2 M_{\pi}}^{\infty} d \mu \mu \frac{\sigma_{i}(\mu)}{\mu^{2}+q^{2}},
$$

where the $\sigma_{i}(\mu)$ are the corresponding mass spectra (spectral functions). Further, $\mathbf{q}$ is the momentum transfer in the centre-of-mass system (c.m.s.), i.e. $\mathbf{q}=\mathbf{p}^{\prime}-\mathbf{p}$, where $\mathbf{p}^{\prime}$ and $\mathbf{p}$ are final and initial nucleon momenta, respectively, and $q \equiv|\mathbf{q}|$. These spectral functions contain the whole dynamics related to the exchanged two-pion system and can be obtained from the potential via [2]

$$
\sigma_{i}(\mu)=\operatorname{Im}\left[W_{i}\left(0^{+}-i \mu\right)\right] .
$$

The spectral function regularization proposed in I suppresses the large $-\mu$ contributions to the integrals Eq. 2.1 via a sharp cut-off

$$
\sigma_{i}(\mu) \rightarrow \theta(\tilde{\Lambda}-\mu) \sigma_{i}(\mu)
$$

and thus regulates the short-distance contributions of the TPE in a natural way.

Next, we briefly review the chiral expansion of the NN potential. The LO potential $V^{(0)}$ is given by OPE and two contact interactions, $V^{(0)}=V_{1 \pi}^{(0)}+V_{\text {cont }}^{(0)}$, with

$$
\begin{aligned}
V_{1 \pi}^{(0)} & =-\left(\frac{g_{A}}{2 F_{\pi}}\right)^{2} \boldsymbol{\tau}_{1} \cdot \boldsymbol{\tau}_{2} \frac{\sigma_{1} \cdot \mathbf{q} \sigma_{2} \cdot \mathbf{q}}{q^{2}+M_{\pi}^{2}}, \\
V_{\text {cont }}^{(0)} & =C_{S}+C_{T} \sigma_{1} \cdot \sigma_{2},
\end{aligned}
$$

where $\sigma(\boldsymbol{\tau})$ are nucleon spin (isospin) matrices. The NLO corrections are due to two-pion exchange

$$
V_{2 \pi}^{(2)}=-\frac{\boldsymbol{\tau}_{1} \cdot \boldsymbol{\tau}_{2}}{384 \pi^{2} F_{\pi}^{4}} L^{\tilde{\Lambda}}(q)\left\{4 M_{\pi}^{2}\left(5 g_{A}^{4}-4 g_{A}^{2}-1\right)\right.
$$

$$
\begin{aligned}
& \left.+q^{2}\left(23 g_{A}^{4}-10 g_{A}^{2}-1\right)+\frac{48 g_{A}^{4} M_{\pi}^{4}}{4 M_{\pi}^{2}+q^{2}}\right\} \\
& -\frac{3 g_{A}^{4}}{64 \pi^{2} F_{\pi}^{4}} L^{\tilde{\Lambda}}(q)\left\{\sigma_{1} \cdot \mathbf{q} \sigma_{2} \cdot \mathbf{q}-q^{2} \sigma_{1} \cdot \sigma_{2}\right\}
\end{aligned}
$$

as well as short-distance contact interactions (local fourfermion terms with two derivatives)

$$
\begin{aligned}
V_{\text {cont. }}^{(2)} & =C_{1} q^{2}+C_{2} k^{2}+\left(C_{3} q^{2}+C_{4} k^{2}\right)\left(\sigma_{1} \cdot \sigma_{2}\right) \\
& +i C_{5} \frac{1}{2}\left(\sigma_{1}+\sigma_{2}\right) \cdot(\mathbf{q} \times \mathbf{k})+C_{6}\left(\mathbf{q} \cdot \sigma_{1}\right)\left(\mathbf{q} \cdot \sigma_{2}\right) \\
& +C_{7}\left(\mathbf{k} \cdot \sigma_{1}\right)\left(\mathbf{k} \cdot \sigma_{2}\right) .
\end{aligned}
$$

Here, $\mathbf{k}=\left(\mathbf{p}^{\prime}+\mathbf{p}\right) / 2$, and the cut-off regularized loop function $L^{\tilde{\Lambda}}(q)$ reads:

$$
\begin{aligned}
L^{\tilde{\Lambda}}(q) & =\theta\left(\tilde{\Lambda}-2 M_{\pi}\right) \frac{\omega}{2 q} \ln \frac{\tilde{\Lambda}^{2} \omega^{2}+q^{2} s^{2}+2 \tilde{\Lambda} q \omega s}{4 M_{\pi}^{2}\left(\tilde{\Lambda}^{2}+q^{2}\right)}, \\
\omega & =\sqrt{q^{2}+4 M_{\pi}^{2}} \\
s & =\sqrt{\tilde{\Lambda}^{2}-4 M_{\pi}^{2}} .
\end{aligned}
$$

The regularized expression for TPE provides an explicit exclusion of the short-range components in the spectrum (i.e. those ones with the range $r<\tilde{\Lambda}^{-1}$ ). Furthermore, at NLO one also has a correction to OPE, It takes the form

$$
V_{1 \pi}^{(2)}=\frac{g_{A} d_{18} M_{\pi}^{2}}{F_{\pi}^{2}} \boldsymbol{\tau}_{1} \cdot \boldsymbol{\tau}_{2} \frac{\left(\sigma_{1} \cdot \mathbf{q}\right)\left(\sigma_{2} \cdot \mathbf{q}\right)}{q^{2}+M_{\pi}^{2}}
$$

where the LEC $d_{18}$ is related to the Goldberger-Treiman discrepancy. Thus, the complete NLO contribution is given by $V^{(2)}=V_{1 \pi}^{(2)}+V_{2 \pi}^{(2)}+V_{\text {cont. }}^{(2)}$. Finally, the cut-off regularized NNLO corrections are represented by the subleading TPE potential. It takes the form:

$$
\begin{aligned}
V_{2 \pi}^{(3)}= & -\frac{3 g_{A}^{2}}{16 \pi F_{\pi}^{4}}\left\{2 M_{\pi}^{2}\left(2 c_{1}-c_{3}\right)-c_{3} q^{2}\right\}\left(2 M_{\pi}^{2}+q^{2}\right) A^{\tilde{\Lambda}}(q) \\
& -\frac{g_{A}^{2}}{32 \pi F_{\pi}^{4}} c_{4}\left(4 M_{\pi}^{2}+q^{2}\right) A^{\tilde{\Lambda}}(q)\left(\boldsymbol{\tau}_{1} \cdot \boldsymbol{\tau}_{2}\right) \\
& \times\left[\left(\sigma_{1} \cdot \mathbf{q}\right)\left(\sigma_{2} \cdot \mathbf{q}\right)-q^{2}\left(\sigma_{1} \cdot \sigma_{2}\right)\right]
\end{aligned}
$$

where

$$
A^{\tilde{\Lambda}}(q)=\theta\left(\tilde{\Lambda}-2 M_{\pi}\right) \frac{1}{2 q} \arctan \frac{q\left(\tilde{\Lambda}-2 M_{\pi}\right)}{q^{2}+2 \tilde{\Lambda} M_{\pi}} .
$$

In what follows, we use these values for the pion decay constant $F_{\pi}$, the pion masses $M_{\pi^{ \pm}}, M_{\pi^{0}}$ and the nucleon mass $m: F_{\pi}=92.4 \mathrm{MeV}, M_{\pi^{ \pm}}=139.570 \mathrm{MeV}$, $M_{\pi^{0}}=134.977 \mathrm{MeV}, m=938.918 \mathrm{MeV}$. In I we adopted the following values for the nucleon axial-vector coupling $g_{A}$ and the LEC $d_{18}: g_{A}=1.26, d_{18}=-0.97 \mathrm{GeV}^{-2}$. Alternatively, one can use the larger value $g_{A}=1.29$ and completely neglect the NLO correction to OPE given in Eq. (2.8) (i.e. set $d_{18}=0$ ). In this work we will adopt 
this second possibility. Notice, however, that such a replacement is not valid in a general case, since the corresponding chiral $g_{A^{-}}$and $d_{18^{-}}$vertices with three and more pion fields are different. For the LECs $c_{1,4}$ we adopt the central values from the $Q^{3}$-analysis of the $\pi N$ system 3]: $c_{1}=-0.81 \mathrm{GeV}^{-1}, c_{4}=3.40 \mathrm{GeV}^{-1}$. For the constant $c_{3}$ the value $c_{3}=-3.40 \mathrm{GeV}^{-1}$ is used, which is on the lower side but still consistent with the results from Ref. 3]: $c_{3}=-4.69 \pm 1.34 \mathrm{GeV}^{-1}$. This value of the LEC $c_{3}$ was found in Ref. 4 to be consistent with empirical NN phase shifts as well as the results from dispersion and conventional meson theories. Further, the same values of the LECs $c_{1,3,4}$ will be used in the upcoming $\mathrm{N}^{3} \mathrm{LO}$ analysis which will be published separately 5 . Interestingly, similar values for the LEC $c_{3}$ have been extracted recently from matching the chiral expansion of the nucleon mass to lattice gauge theory results at pion masses between 500 and $800 \mathrm{MeV}$, see 6 for more details. Notice that at NNLO in the chiral expansion of the NN potential one could, in principle, use the values of the LECs obtained in the $Q^{2}$-analysis of $\pi N$ scattering. This certain freedom in choosing the values of $c_{i}$ results in some uncertainty in observables which might be viewed as an estimation of some higher order effects. We would also like to remark that a new $n p$ and $p p$ partial wave analysis of the Nijmegen group 7. leads to $c_{3}=-4.78 \pm 0.10 \mathrm{GeV}^{-1}$ and $c_{4}=3.96 \pm 0.22$ $\mathrm{GeV}^{-1}$ using $c_{1}=-0.76 \mathrm{GeV}^{-1}$ as input. These values of the LECs $c_{3,4}$ are close to the ones of Ref. 3 .

Using this potential, one can now generate bound and scattering states. For that, consider the partial-wave projected Lippmann-Schwinger (LS) equation for the NN Tmatrix:

$$
\begin{aligned}
T_{l, l^{\prime}}^{s j}\left(p^{\prime}, p\right)= & V_{l, l^{\prime}}^{s j}\left(p^{\prime}, p\right)+\sum_{l^{\prime \prime}} \int \frac{d^{3} p^{\prime \prime}}{(2 \pi)^{3}} V_{l, l^{\prime \prime}}^{s j}\left(p^{\prime}, p^{\prime \prime}\right) \\
& \times \frac{m}{p^{2}-\left(p^{\prime \prime}\right)^{2}+i \epsilon} T_{l^{\prime \prime}, l^{\prime}}^{s j}\left(p^{\prime \prime}, p\right)
\end{aligned}
$$

where $V=V^{(0)}+V^{(2)}+V^{(3)}, m$ is the nucleon mass. The on-shell $\mathrm{S}$ - and $\mathrm{T}$-matrices are related via

$$
S_{l, l^{\prime}}^{s j}(p, p)=\delta_{l l^{\prime}}-\frac{i}{8 \pi^{2}} p m T_{l, l^{\prime}}^{s j}(p, p) .
$$

Contrary to our previous work I, where we have been interested only in peripheral NN scattering and thus calculated the $\mathrm{T}$-matrix perturbatively (i.e. keeping only the Born term in Eq. (2.11), we now have to solve the LS equation non-perturbatively.

Although we have regularized the TPE contributions by cutting off the large--mass components in the spectrum (or, equivalently, by explicitly shifting the corresponding short-distance components to contact terms), the resulting potential still behaves incorrectly at large $q$ (or equivalently at small $r$ ). The effective potential is valid for small values of the momentum transfer $q$ and becomes meaningless for $q \gtrsim \Lambda_{\chi}$. Moreover, since the potential $V$ grows with increasing momenta $q$, the LS equation (2.11) is ultraviolet divergent and needs to be regularized. Following the standard procedure, see e.g. [8], we introduce an additional cut-off in the LS equation by multiplying the potential $V\left(\mathbf{p}, \mathbf{p}^{\prime}\right)$ with a regulator function $f^{\Lambda}$,

$$
V\left(\mathbf{p}, \mathbf{p}^{\prime}\right) \rightarrow f^{\Lambda}(p) V\left(\mathbf{p}, \mathbf{p}^{\prime}\right) f^{\Lambda}\left(p^{\prime}\right) .
$$

In what follows, we use the exponential regulator function

$$
f^{\Lambda}\left(p^{\prime}\right)=\exp \left[-p^{6} / \Lambda^{6}\right]
$$

Certainly, both cut-offs $\tilde{\Lambda}$ and $\Lambda$ are introduced in order to remove high-momentum components of the interacting nucleon and pion fields. The physical meaning and the implementation of the cut-offs $\tilde{\Lambda}$ and $\Lambda$ is, however, quite different from each other: while the first one removes the short-distance portion of the TPE nuclear force, the second one guarantees that the high-momentum nucleon states do not contribute to the scattering process. One advantage of the method proposed in I is that one can (but does not have to) choose similar procedures for regulating the spectral functions and the LS equation. For further discussion of the role and optimal choice of the cut-off $\Lambda$ in the LS equation the reader is referred to Refs. 9.10 .

In what follows we will vary the cut-off $\Lambda$ in the LS equation in the range $450 \ldots 600 \mathrm{MeV}$ at NLO and $450 \ldots$ $650 \mathrm{MeV}$ at NNLO, which is a significantly larger range than in Ref. [1]. The cut-off $\tilde{\Lambda}$ is varied independently in the range $500 \ldots 700 \mathrm{MeV}$, which is consistent with the variation of $\Lambda$. Notice that in principle, more elegant regularization prescriptions, like e.g. lattice regularization, would allow to regularize pion loop integrals and the Lippmann-Schwinger equation in the same way without introducing two independent scales $\Lambda$ and $\tilde{\Lambda}$.

\section{Results}

For any choice of the cut-offs $\Lambda$ and $\tilde{\Lambda}$, the LECs $C_{S, T}$ and $C_{1 \ldots 7}$ are fixed from a fit to the $n p \mathrm{~S}-$ and $\mathrm{P}$-waves and the mixing parameter $\epsilon_{1}$ for laboratory energies below 100 $\mathrm{MeV}$. This is the same procedure as used in Ref. 8. In the following, we display and discuss our predictions for the phase shifts at higher energies and higher angular momenta as well as for various deuteron properties. We also discuss the values of the pertinent low-energy constants (LECs) and their interpretation in terms of resonance saturation along the lines of Ref. [12.

Before presenting the results we would like to make several comments concerning the theoretical uncertainty and our way of estimating it. When performing calculations within chiral EFT up to a certain order $\mathcal{O}\left(Q^{n} / \lambda^{n}\right)$, where $Q \sim M_{\pi}$ refers to a generic low-momentum scale (soft scale) and $\lambda$ to the scale at which new physics appears (hard scale), the theoretical uncertainty results from neglecting the higher order terms and is, in general, expected to be of the order $\sim(Q / \lambda)^{n+1}$. In particular, one expects for the uncertainty of a scattering observable at c.m.s. momentum $p$ to be of the order $\sim\left(\max \left[p, M_{\pi}\right] / \lambda\right)^{n+1}$. One should keep in mind that while the hard scale $\lambda$ is governed by $\Lambda_{\chi} \sim M_{\rho}$ in perturbative calculations in the $\pi \pi$ and $\pi N$ sectors, where dimensional regularization is 

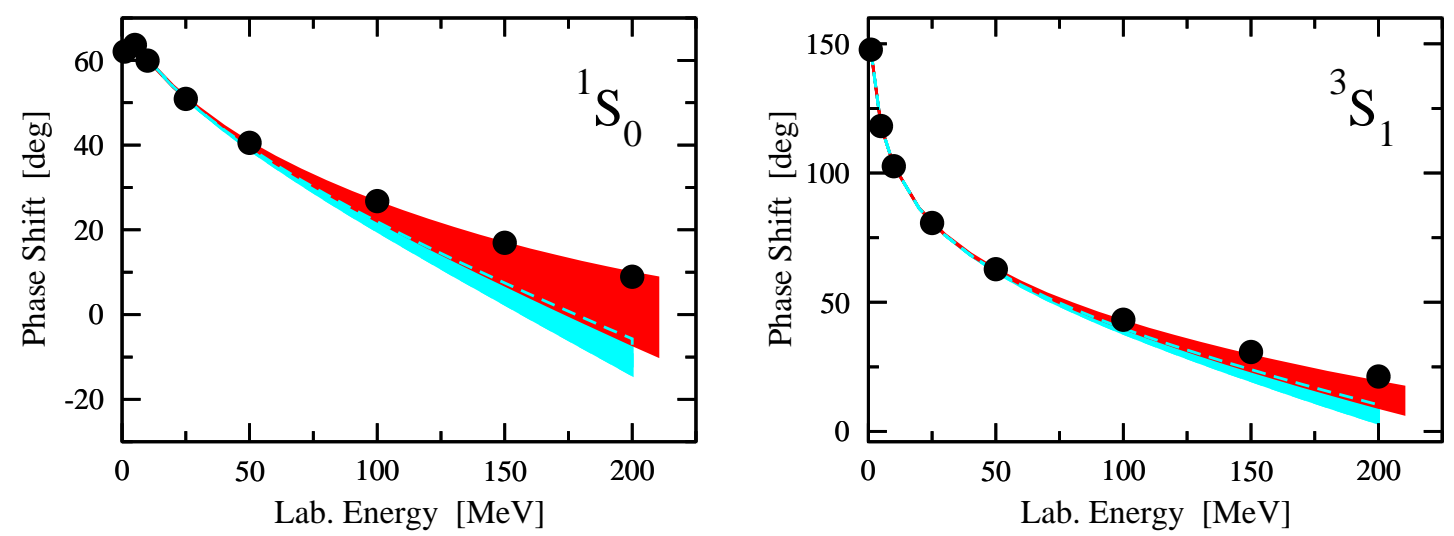

Fig. 1. S-wave NN phase shifts versus the nucleon laboratory energy. The light (dark) shaded band shows the NLO (NNLO) predictions with CR chiral TPE. The cut-off $\Lambda$ in the Lippmann-Schwinger equation is varied in the range $\Lambda=450 \ldots 600 \mathrm{MeV}$ at $\mathrm{NLO}$ and $\Lambda=450 \ldots 650$ at NNLO. The cut-off $\tilde{\Lambda}$ in the pion loops is varied independently in the range $\tilde{\Lambda}=500 \ldots 700 \mathrm{MeV}$ at both NLO and NNLO. The filled circles depict the Nijmegen phase shift analysis (PSA) results [13.

usually applied and no finite momentum space cut-off is introduced, $\lambda \sim \min \left[\Lambda, \tilde{\Lambda}, M_{\rho}\right]=450 \mathrm{MeV}$ should be adopted in our case.

Cut-off variation became a common practice to estimate the uncertainty and to check consistency of nonperturbative EFT calculations of few-nucleon systems. Low-energy observables should not depend on the cutoff value if all terms in the EFT expansion are included. In practice, however, calculations are performed at a finite order, so that some (small) residual dependence of observables on the cut-off remains. One, in general, expects that this cut-off dependence gets weaker when higher order terms are included. Thus, at first sight one expects narrower bands for scattering observables at NNLO than at NLO for the same variation of the cut-off. This, however, does not hold true for the following reason: the cut-off dependence at both NLO and NNLO has to be compensated by inclusion of the contact interactions (counter terms) of the order $\mathcal{O}\left(Q^{4} / \lambda^{4}\right)$ and higher. The contact interactions appear only at even orders $\mathcal{O}\left(Q^{2 l} / \lambda^{2 l}\right)$ in the lowmomentum expansion while pion exchanges contribute, in general, at both even and odd orders. Since the same contact terms enter the expression for the effective potential at NLO and NNLO, similar cut-off dependence for observables should be expected at these orders. Variation of the cut-off does probably not provide an appropriate estimation of theoretical uncertainty at NLO since it does not rely on missing $\mathcal{O}\left(Q^{3} / \lambda^{3}\right)$-terms, but only on $\mathcal{O}\left(Q^{4} / \lambda^{4}\right)$ corrections. Notice further that we were only able to vary the cut-off $\Lambda$ in the LS equation at NLO in the smaller range compared to NNLO, which partially explains why the NLO bands in many cases even turn out to be narrower than NNLO ones. Another reason for that behavior has already been discussed in I: variation of the spectral function cut-off $\tilde{\Lambda}$ has only small effect at NLO, since the leading TPE in most cases provides a very small correc- tion to the LO potential. The corrections from subleading TPE at NNLO are significantly larger in magnitude (at large $q$ ), which leads to larger variation of the potential associated with the spectral function regularization, see I for more details.

\subsection{S-waves}

The phase shifts in the ${ }^{1} S_{0}$ and ${ }^{3} S_{1}$ partial waves are shown in Fig. [1 One observes a clear improvement when going from NLO to NNLO and the description of both phases is satisfactory up to the considered energy $E_{\text {lab }}=$ $200 \mathrm{MeV}$. One should keep in mind that this improvement is entirely due to inclusion of the subleading TPE potential, since no new contact operators arise at NNLO and thus the number of adjustable parameters is the same at NLO and NNLO.

As expected, the bands at NLO and NNLO are roughly of the same width. In the ${ }^{1} S_{0}$-channel, the band at NNLO is even wider than at NLO, the latter, however, does not properly describe the data at larger energies. We remind the reader at this point that the bands at NLO underestimate the uncertainty of the theory at this order. It is comforting to see that the bands at NLO and NNLO overlap and that the Nijmegen values of the S-wave phase shifts are reproduced at NNLO within the theoretical uncertainty.

It is also of interest to consider the scattering length and effective range parameters. The effective range expansion in the $\mathrm{S}$-waves takes the form:

$$
p \cot (\delta)=-\frac{1}{a}+\frac{1}{2} r p^{2}+v_{2} p^{4}+v_{3} p^{6}+v_{4} p^{8}+\mathcal{O}\left(p^{10}\right),
$$

where $p$ is the nucleon centre-of-mass momentum, $a$ is the scattering length, $r$ is the effective range and the $v_{i}$ are the 


\begin{tabular}{||l||c|c|c||c||}
\hline \hline & NLO, CR & NNLO, CR & NNLO, DR & Nijmegen PSA \\
\hline \hline$a[\mathrm{fm}]$ & $-23.447 \ldots-23.522$ & $-23.497 \ldots-23.689$ & -23.936 & -23.739 \\
$r[\mathrm{fm}]$ & $2.60 \ldots 2.62$ & $2.62 \ldots 2.67$ & 2.73 & 2.68 \\
$v_{2}\left[\mathrm{fm}^{3}\right]$ & $-0.46 \ldots-0.47$ & $-0.48 \ldots-0.52$ & -0.46 & -0.48 \\
$v_{3}\left[\mathrm{fm}^{5}\right]$ & $4.3 \ldots 4.4$ & $4.0 \ldots 4.2$ & 3.8 & 4.0 \\
$v_{4}\left[\mathrm{fm}^{7}\right]$ & $-20.7 \ldots-21.0$ & $-19.9 \ldots-20.5$ & -19.1 & -20.0 \\
\hline \hline
\end{tabular}

Table 1. Scattering length and range parameters for the ${ }^{1} S_{0}$ partial wave using the CR NLO and NNLO potential compared to the DR results (with $\Lambda=1000 \mathrm{MeV}$ ) and to the Nijmegen phase shift analysis (PSA). The values $v_{2,3,4}$ are based on the $n p$ Nijm II potential and the values of the scattering length and the effective range are from Ref. [15].

shape parameters. The coefficients in the effective range expansion are governed by the long-distance physics associated with exchange of pions and thus serve as a good testing ground for the convergence of the chiral expansion 14. To be specific, let us consider the ${ }^{1} S_{0}$ partial wave. For each value of the cut-off $\Lambda$ in the LippmannSchwinger equation one has to determine the values of the LECs $\tilde{C}_{{ }_{S}}, C_{1} S_{0}$, which accompany the (partial-wave projected) contact operators without and with two derivatives, respectively. These LECs can be fixed, for instance, from the first two coefficients in the effective range expansion, i.e. from $a$ and $r$, so that predictions for the $v_{i}$ 's can be made. Alternatively, one can fix them from a fit to the phase shift at low energy and then calculate all coefficients $a, r$ and $v_{i}$. We will adopt this second method in what follows.

In Table1we present our results for the effective range coefficients in the ${ }^{1} S_{0}$ channel. Already the NLO results are in a reasonable agreement with the data (as given by the Nijmegen PSA). At NNLO we find an improved description for all effective range coefficients. The scattering length and effective range are still not exactly reproduced at this order if a global fit to data is performed. The uncertainty for the scattering length and effective range at NNLO resulting from variation of the cut-offs $\Lambda$ and $\tilde{\Lambda}$ is of the order of 0.2 and $0.05 \mathrm{fm}$, respectively, which is much larger than the deviations from the experimental values $(\sim 0.05$ and $\sim 0.01 \mathrm{fm})$. The shape coefficients are reproduced at NNLO within the theoretical uncertainty.

We also show the result based on the DR NNLO potential with the cut-off $\Lambda$ in the Lippmann-Schwinger equation taken as $\Lambda=1000 \mathrm{MeV}$. Notice that there are two unphysical deeply bound states in each of the ${ }^{1} S_{0}$ and ${ }^{3} S_{1}-{ }^{3} D_{1}$ channels in that case. The results for the effective range coefficients are a bit less precise than the ones obtained with CR TPE.

Our results for the effective range parameters in the ${ }^{3} S_{1}$ channel are shown in Table 2 Similarly to the previously considered case, we observe at NNLO an improved description for all coefficients. The predicted values of the scattering length and effective range are close to the experimental ones (within $0.1 \%$ and $1 \%$, respectively). In the case of the ${ }^{3} S_{1}$ channel, the DR results with $\Lambda=1000$ $\mathrm{MeV}$ are slightly more precise than the $\mathrm{CR}$ ones.

\subsection{P-waves}

Our results for the $\mathrm{P}$-waves and the mixing angle $\epsilon_{1}$ are shown in Fig. 2. While the ${ }^{1} P_{1},{ }^{3} P_{1}$ and ${ }^{3} P_{2}$ phase shifts are visibly improved at NNLO compared to the NLO results, the NNLO results for the ${ }^{3} P_{0}$ phase shift disagree with the data for energies higher than $E_{\text {lab }} \sim 100 \mathrm{MeV}$, where the NLO results are in a better agreement. At the moment, we do not have an explanation for this disagreement with the data in the ${ }^{3} P_{0}$ channel. We have checked that this is corrected at $\mathrm{N}^{3} \mathrm{LO}$, where a new counter term appears in that partial wave. As in the case of $\mathrm{S}$-waves, the bands at NLO and NNLO are of a similar width. The theoretical uncertainty in the ${ }^{1} P_{1}$ channel is probably underestimated by the variation of the cut-offs $\Lambda, \tilde{\Lambda}$.

In general, our NNLO results for the phase shifts based upon CR TPE look similar to the ones of Ref. 8, where dimensional regularization has been used to calculate pion loops. In the latter case, the TPE potential shows unphysically strong attraction at intermediate and short distances, see 11 1] for more details. Although a reasonably good description is possible with DR TPE at NNLO, as documented in [8, unphysical deeply bound states arise in D- and lower partial waves and one has serious problems with the convergence of the chiral expansion. In particular, changing the value of the cut-off in the LippmannSchwinger equation clearly leads to a strong variation of the D-wave phase shifts, where the potential still turns out to be very strong. The problem with the convergence is manifest, since there are no counter terms to compensate this cut-off dependence at NNLO. Using spectral function regularization in the pion loops as discussed in I we are now able to describe the data equally well as with the DR version and in addition: 


\begin{tabular}{||l||c|c|c||c||}
\hline \hline & NLO, CR & NNLO, CR & NNLO, DR & Nijmegen PSA \\
\hline \hline$a[\mathrm{fm}]$ & $5.429 \ldots 5.433$ & $5.424 \ldots 5.430$ & 5.416 & 5.420 \\
$r[\mathrm{fm}]$ & $1.710 \ldots 1.722$ & $1.725 \ldots 1.735$ & 1.756 & 1.753 \\
$v_{2}\left[\mathrm{fm}^{3}\right]$ & $0.06 \ldots 0.07$ & $0.04 \ldots 0.05$ & 0.04 & 0.04 \\
$v_{3}\left[\mathrm{fm}^{5}\right]$ & $0.77 \ldots 0.81$ & $0.71 \ldots 0.76$ & 0.67 & 0.67 \\
$v_{4}\left[\mathrm{fm}^{7}\right]$ & $-4.3 \ldots-4.4$ & $-4.1 \ldots-4.3$ & -4.1 & -4.0 \\
\hline \hline
\end{tabular}

Table 2. Scattering length and range parameters for the ${ }^{3} S_{1}$ partial wave using the CR NLO and NNLO potential compared to the DR results and to the Nijmegen PSA [16].

- one can use the same values for the cut-off in the Lippmann-Schwinger equation in the LO and NLO versions, which are slightly below the mass of the $\rho$ meson,

- one does not have spurious deeply bound states,

- one has a convergent expansion.

Notice that we use here the same values for the LECs $c_{1,4}$ as in Ref. [8] and somewhat smaller in magnitude value for the LEC $c_{3}$. Further, we do not include the (incomplete) set of leading relativistic $(1 / m)$ corrections as done in that paper.

\subsection{D- and selected higher partial waves}

The results for $\mathrm{D}$-waves have already been analyzed in I making use of the Born-approximation. In Fig. [3] we show our results for D-waves obtained by solving the LS equation. As expected, the results are quite similar to the ones found in I. The small differences like e.g. slightly different shape of the ${ }^{3} D_{2}$ and $\epsilon_{2}$ phase shifts arise due to introduction of the exponential regulator function in Eq. (2.13), the exact solution of the LS equation as well as due to slightly different value of the LEC $c_{3}$ adopted in the present work. Similar results for the phase shifts in the present work and in I confirm the high accuracy of the Born approximation in these channels.

As in the previously considered channels, the bands at NLO and NNLO are of a comparable width (with exception of the ${ }^{1} D_{2}$ partial wave), and the NNLO results are in a better agreement with the data.

We show in Fig. 4 selected higher partial waves, which also display a very similar behavior to the one observed in I. We remind the reader that a significant disagreement with the data in the ${ }^{3} G_{5}$ channel at both NLO and NNLO should not be considered as a problem because of the exceptionally small value of the phase shift in this particular channel (more than 10 times smaller in magnitude compared to other $\mathrm{G}$-waves).

\subsection{Deuteron properties}

We now turn to the bound state properties. We stress that we do not use the deuteron binding energy as a fit parameter as it is frequently done but rather adopt the same parameters as obtained in the fit to the low phases. In Table 3 we collect the resulting deuteron properties, in comparison to the DR results with $\Lambda=1000 \mathrm{MeV}$ and the CD-Bonn potential. First, we note a clear improvement when going from NLO to NNLO. In particular the predicted binding energy deviates by $1 \%-1.5 \%$ at NNLO to be compared with $\sim 2 \%-2.5 \%$ deviation at NLO. Also visibly improved are the root-mean-square matter radius $r_{d}$ and the asymptotic S-wave normalization strength $A_{S}$. For $r_{d}$, a surprisingly good agreement with the data is observed: the NLO (NNLO) prediction deviate from the experimental value by less than $0.35 \%$ (0.25\%). Deviations from the data for the asymptotic $\mathrm{S}$-wave normalization strength $A_{S}$ of $\sim 2 \%(\sim 1 \%)$ at NLO (NNLO) are of the expected size: for a typical deuteron observable one expects the uncertainty at NLO (NNLO) to be of the order $\sim M_{\pi}^{3} /(450 \mathrm{MeV})^{3} \sim 3 \%\left(\sim M_{\pi}^{4} /(450 \mathrm{MeV})^{4} \sim 1 \%\right)$. The quadrupole moment $Q_{d}$ is the only deuteron property for which our predictions seem to disagree with the observed value by somewhat larger amount than expected, namely by $\sim 3.8 \%-4.5 \%$ at NLO and $\sim 3.8 \%-5.2 \%$ at NNLO. It is, however, well known that $Q_{d}$ is rather sensitive to short-range physics, see e.g. 17. The deuteron quadrupole moment also turns out to be underpredicted by $\sim 4 \%$ in modern potential model calculations. Notice further that similar values for $Q_{d}$ where found in Ref. 18 where DR was used and the pertinent LECs were fine-tuned to the deuteron binding energy.

The DR NNLO predictions shown in Table 3 are in a better (somewhat worse) agreement with the data for $A_{S}$ $\left(Q_{d}\right)$ and of the same quality for the other observables as the CR NNLO results.

\subsection{Low-energy constants and resonance saturation}

We are now in the position to confront the LECs determined from chiral effective field theory with the highly 

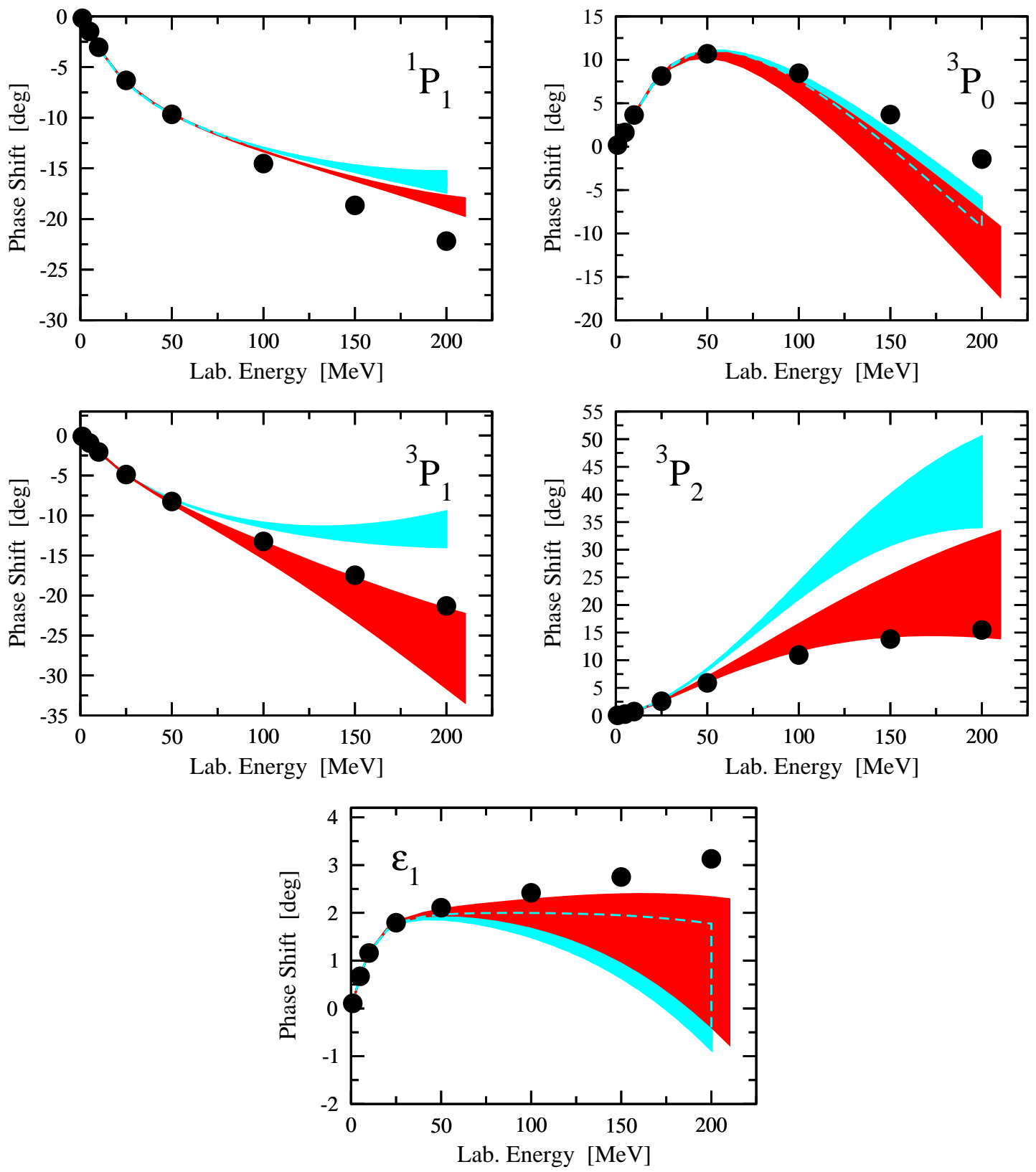

Fig. 2. $\mathrm{P}$-wave $\mathrm{NN}$ phase shifts and mixing angle $\epsilon_{1}$ versus the nucleon laboratory energy. For notation see Fig. 1

successful phenomenological/meson exchange models of the nuclear force following the lines of Ref. [12. In this work we will consider the Bonn-B [19] and Nijmegen 93 20] potentials, which are genuine one-boson-exchange (OBE) models. In these models the long range part of the interaction is given by OPE (including a pion-nucleon form factor) whereas shorter distance physics is expressed as a sum over heavier mesons exchange contributions:

$$
V_{\mathrm{NN}}=V_{\pi}+\sum_{M=\sigma, \rho, \ldots} V_{M}
$$

Here some mesons can be linked to real resonances (like e.g. the $\rho$-meson) or are parameterizations of certain physical effects, e.g. the light scalar-isoscalar $\sigma$-meson is needed to supply the intermediate range attraction (but it is not a resonance). The corresponding meson-nucleon vertices are given in terms of one (or two) coupling constant(s) and corresponding form factor(s), characterized by some cut-off scale. These form factors are needed to regularize the potential at small distances (large momenta) but they should not be given a physical interpretation. As depicted in Fig $[$ for nucleon momentum transfer below 

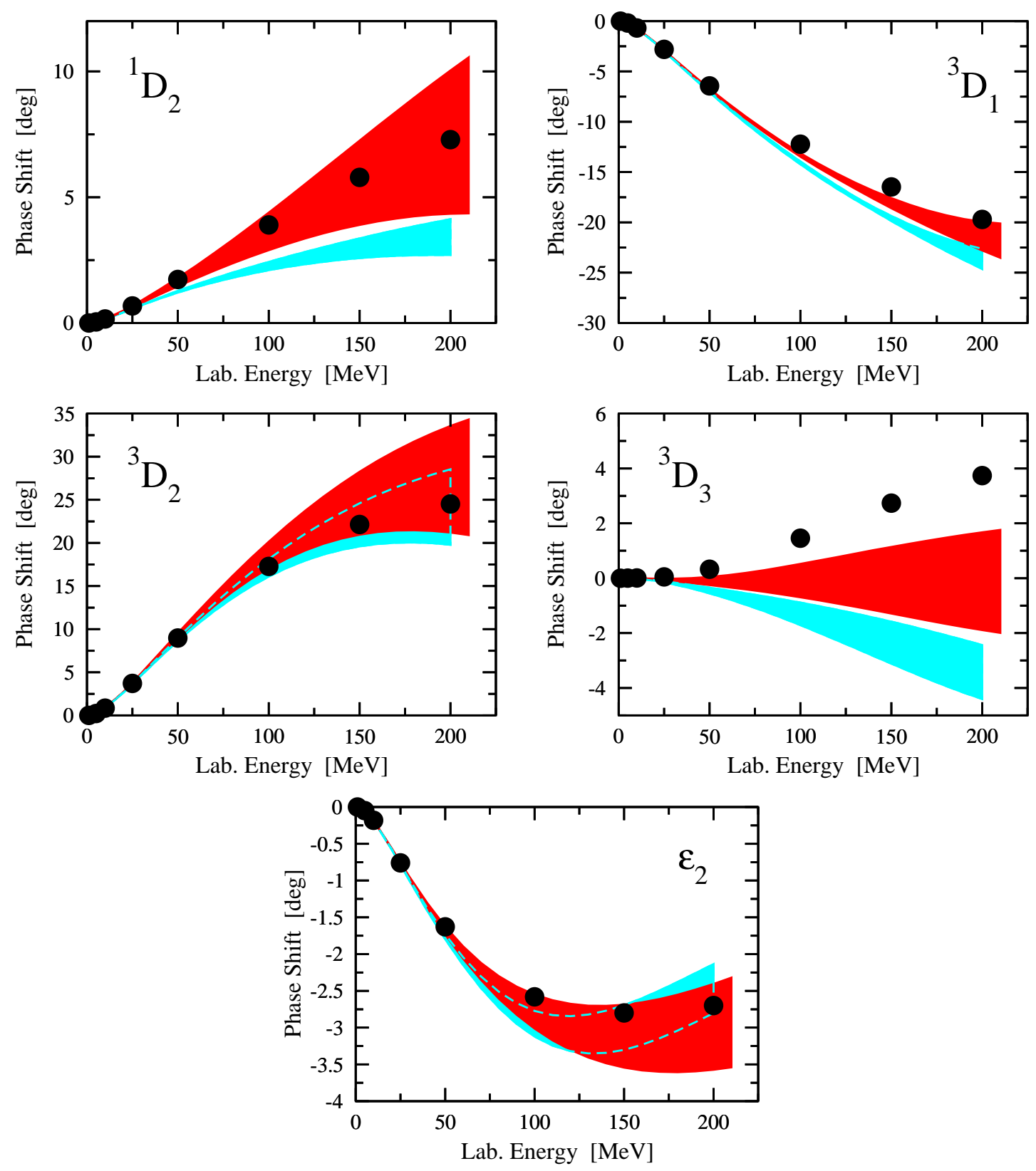

Fig. 3. D-wave NN phase shifts and mixing angle $\epsilon_{2}$ versus the nucleon laboratory energy. For notation see Fig. 1

the masses of the exchanged mesons, one can interpret such exchange diagrams as a sum of local operators with increasing number of derivatives (momentum insertions). This is explained in detail in Ref. 12. In that work we power expanded the short-range part of different phenomenological potential models and compared the resulting contact operators with the ones in the EFT approach. The latter have to be corrected by adding the corresponding power expanded TPE contributions, which are not present in the phenomenological models. We have then demonstrated explicitly that the values of the LECs $C_{i}$ determined from various phenomenological OBE models are close to the values found in EFT at NLO and NNLO. The TPE contributions have been calculated in [12] using dimensional regularization. In that work we have restricted ourselves to the NNLO version with numerically small values of the LECs $c_{3,4}, c_{3}=-1.15, c_{4}=1.20 \mathrm{GeV}^{-1}$, which are not consistent with the $\pi N$ system. Using the values of these LECs obtained from the $\pi N$ system in the TPE potential calculated with dimensional regularization (or equivalent schemes) leads, as explained before, to convergence problems due to the strong attraction in the central part of the potential. In particular, the $\mathrm{D}$-wave phase shifts turn out to be strongly cut-off dependent. Further, 

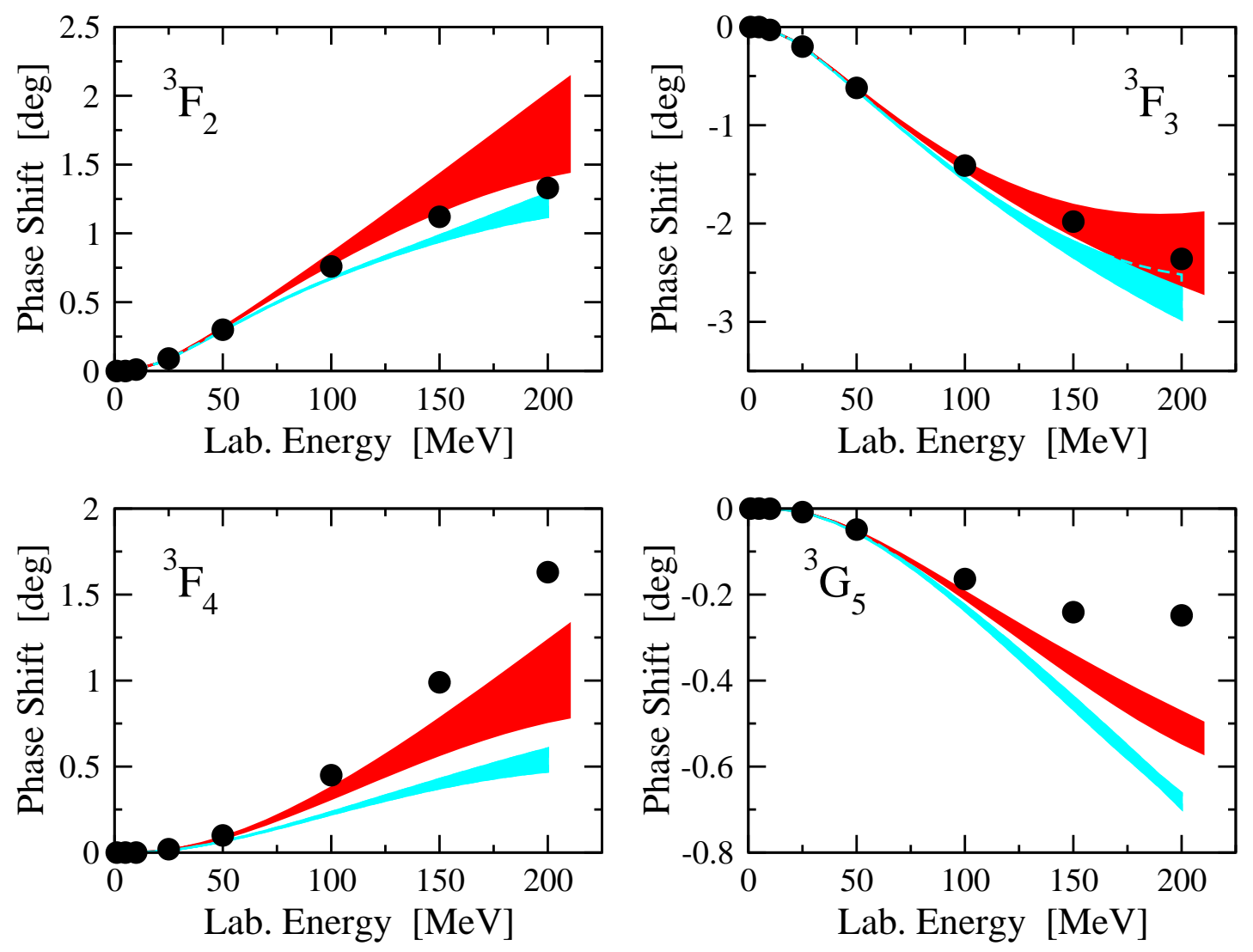

Fig. 4. Selected peripheral NN phase shifts versus the nucleon laboratory energy. For notation see Fig. 1]

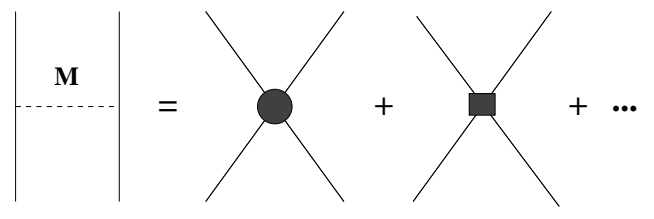

Fig. 5. Expansion of a meson exchange diagram in terms of local four-nucleon operators. The dashed and solid lines denote the meson $M=\rho, \sigma, \omega, \ldots$ and the nucleons, respectively. The blob and the square denote insertions with zero and two derivatives, in order. The ellipses stands for operators with more derivatives.

unphysical deeply bound states arise in low partial waves. Obviously, no resonance saturation can be established for this NNLO version of the potential, which is strongly nonphase-equivalent to the phenomenological OBE models. For example, the LECs $C_{i}$ in this NNLO version are typically several times larger in magnitude and differ very much from the values at NLO.

We will now demonstrate how resonance saturation works for the chiral NN forces at NLO and NNLO introduced above, where the new spectral function regularization has been used to derive the TPE contributions. Differently to Ref. 12, all LECs $c_{i}$ are now consistent with $\pi N$ scattering. In Table 4 we compare the values of the LECs $C_{i}$ at NLO and NNLO with the ones re- sulting from the OBE models as explained before. We remind the reader that the contribution from chiral TPE should be accounted for properly in order to allow for a meaningful comparison with the OBE models. To achieve that we power expand the chiral TPE at NLO and NNLO and identify the corresponding contributions to the LECs, which are given analytically in appendix A Notice that differently to 12 these contributions depend now on the spectral function cut-off $\tilde{\Lambda}$. The second and third columns in Table 4 show the corresponding numerical results at NLO and NNLO for the central value $\tilde{\Lambda}=600 \mathrm{MeV}$, respectively. The indicated uncertainty refers to the cutoff variation $\tilde{\Lambda}=500 \ldots 700 \mathrm{MeV}$. The fourth and fifth columns contain the values of the LECs $C_{i}$ at $\mathrm{NLO}$ and NNLO, where the just discussed contributions from TPE have already been added. The numbers are presented for our central values of $\Lambda$ and $\tilde{\Lambda}, \Lambda=550 \mathrm{MeV}$ and $\tilde{\Lambda}=$ $600 \mathrm{MeV}$ and the uncertainties refer to variations $\tilde{\Lambda}=$ $500 \ldots 700 \mathrm{MeV}$ and $\Lambda=450 \ldots 600 \mathrm{MeV}(\Lambda=450 \ldots 650$ $\mathrm{MeV})$ at $\mathrm{NLO}(\mathrm{NNLO})$. Notice that the uncertainties due to the variation of $\Lambda$ and $\tilde{\Lambda}$ are very large in several cases: for $\tilde{C}_{1 S 0}$ and $C_{1 S 0}$ at NLO and for $C_{3 P 0}$ and $C_{3 P 1}$ at NNLO. Such a strong variation in the values of the LECs arises when the cut-off $\Lambda$ in the LS equation becomes too large and one leaves the plateau-region for the corresponding $\operatorname{LEC} C_{i}(\Lambda)$. This situation is exemplified in Fig 6] where we show the $\Lambda$-dependence of the LEC $C_{3 P 1}$ 


\begin{tabular}{||l||c|c|c||c||c||}
\hline \hline & NLO, CR & NNLO, CR & NNLO, DR & CD-Bonn & Exp. \\
\hline \hline$E_{d}[\mathrm{MeV}]$ & $-2.171 \ldots-2.186$ & $-2.189 \ldots-2.202$ & -2.230 & $-2.225^{\star}$ & -2.225 \\
$Q_{d}\left[\mathrm{fm}^{2}\right]$ & $0.273 \ldots 0.275$ & $0.271 \ldots 0.275$ & 0.270 & 0.270 & 0.286 \\
$\eta$ & $0.0256 \ldots 0.0257$ & $0.0255 \ldots 0.0256$ & 0.0257 & 0.0255 & 0.0256 \\
$r_{d}[\mathrm{fm}]$ & $1.973 \ldots 1.974$ & $1.970 \ldots 1.972$ & 1.970 & 1.966 & 1.967 \\
$A_{S}\left[\mathrm{fm}^{-1 / 2}\right]$ & $0.868 \ldots 0.873$ & $0.874 \ldots 0.879$ & 0.886 & 0.885 & 0.885 \\
$P_{D}[\%]$ & $3.46 \ldots 4.29$ & $3.53 \ldots 4.93$ & 6.71 & 4.83 & - \\
\hline \hline
\end{tabular}

Table 3. Deuteron properties derived from the CR chiral potential at NLO and NNLO compared to the DR NNLO results of [8, one "realistic" potential and the data. Here, $E_{d}$ is the binding energy, $Q_{d}$ the quadrupole moment, $\eta$ the asymptotic $D / S$ ratio, $r_{d}$ the root-mean-square matter radius, $A_{S}$ the strength of the asymptotic S-wave normalization and $P_{D}$ the D-state probability. ${ }^{\star}$ denotes an input quantity.

\begin{tabular}{||l||c|c||c|c||c|c||}
\hline \hline LEC & TPE (NLO) & TPE (NNLO) & $C_{i}(\mathrm{NNLO})$ & $C_{i}(\mathrm{NNLO})$ & Bonn B & Nijm-93 \\
\hline \hline$\tilde{C}_{1 S 0}$ & $-0.004_{-0.001}^{+0.000}$ & $-0.004_{-0.001}^{+0.000}$ & $-0.117_{-0.042}^{+2.271}$ & $-0.158_{-0.004}^{+0.178}$ & -0.117 & -0.061 \\
$C_{1 S 0}$ & $-0.570_{-0.022}^{+0.036}$ & $-0.443_{-0.057}^{+0.078}$ & $1.294_{-0.322}^{+2.873}$ & $1.213_{-0.084}^{+0.408}$ & 1.276 & 1.426 \\
$\tilde{C}_{3 S 1}$ & $0.013_{-0.000}^{+0.001}$ & $-0.004_{-0.001}^{+0.000}$ & $-0.135_{-0.021}^{+0.025}$ & $-0.137_{-0.027}^{+0.017}$ & -0.101 & -0.014 \\
$C_{3 S 1}$ & $0.638_{-0.044}^{+0.025}$ & $-0.443_{-0.057}^{+0.078}$ & $0.231_{-0.007}^{+0.112}$ & $0.523_{-0.039}^{+0.197}$ & 0.660 & 0.940 \\
$C_{\epsilon 1}$ & $-0.190_{-0.006}^{+0.012}$ & $0.205_{-0.035}^{+0.024}$ & $-0.325_{-0.036}^{+0.000}$ & $-0.395_{-0.072}^{+0.007}$ & -0.410 & -0.343 \\
$C_{1 P 1}$ & $-0.067_{-0.005}^{+0.007}$ & $-0.090_{-0.009}^{+0.013}$ & $0.146_{-0.010}^{+0.005}$ & $0.126_{-0.017}^{+0.023}$ & 0.454 & 0.119 \\
$C_{3 P 0}$ & $-0.425_{-0.014}^{+0.025}$ & $0.006_{-0.003}^{+0.003}$ & $0.923_{-0.103}^{+0.142}$ & $0.920_{-0.109}^{+1.063}$ & 0.921 & 0.802 \\
$C_{3 P 1}$ & $0.246_{-0.016}^{+0.009}$ & $0.247_{-0.044}^{+0.032}$ & $-0.260_{-0.005}^{+0.003}$ & $-0.108_{-0.176}^{+2.364}$ & -0.075 & -0.197 \\
$C_{3 P 2}$ & $-0.022_{-0.000}^{+0.000}$ & $0.151_{-0.028}^{+0.020}$ & $-0.262_{-0.073}^{+0.032}$ & $-0.421_{-0.052}^{+0.074}$ & -0.396 & -0.467 \\
\hline \hline
\end{tabular}

Table 4. The LECs $C_{i}$ at NLO and NNLO compared with the results from the Bonn B and Nijmegen 93 OBE potential models. Also shown are contributions from chiral TPE as explained in text. The $\tilde{C}_{i}$ are in $10^{4} \mathrm{GeV}^{-2}$ and the $C_{i}$ in $10^{4} \mathrm{GeV}^{-4}$.

at NNLO for $\tilde{\Lambda}=600 \mathrm{MeV}$. The $\Lambda$-dependence of $C_{3 P 1}$ is similar to the one of the three-nucleon force observed in Ref. [21. ${ }^{1}$ The first branch in Fig. [6] (for $\Lambda \lesssim 730 \mathrm{MeV}$ ) corresponds to the case of no deeply bound states. For larger values of $\Lambda$ unphysical deeply bound states arise. In that case the situation is similar to the DR NNLO analysis of Ref. 8]. Clearly, it only makes sense to discuss resonance saturation of the $C_{i}$ 's in the plateau-region of the

\footnotetext{
1 The long-range part of the potential in that reference behaves like $1 / r^{2}$ at short distances, while the CR chiral TPE at NNLO is even more singular and behaves like $1 / r^{5}$, see I.
}

first branch, where they only change modestly and where the effective potential is at least not strongly non-phaseequivalent to the OBE models. Notice that the strong variation of the LECs with the cut-off did not occur in Ref. 12] simply because $\Lambda$ was varied in a much smaller range and never left the plateau-region.

The last two columns in Table 4 show the LECs as predicted by resonance saturation based upon the Nijmegen 93 and Bonn B potential models. As in Ref. [12, we observe a remarkable agreement between the LEC values 


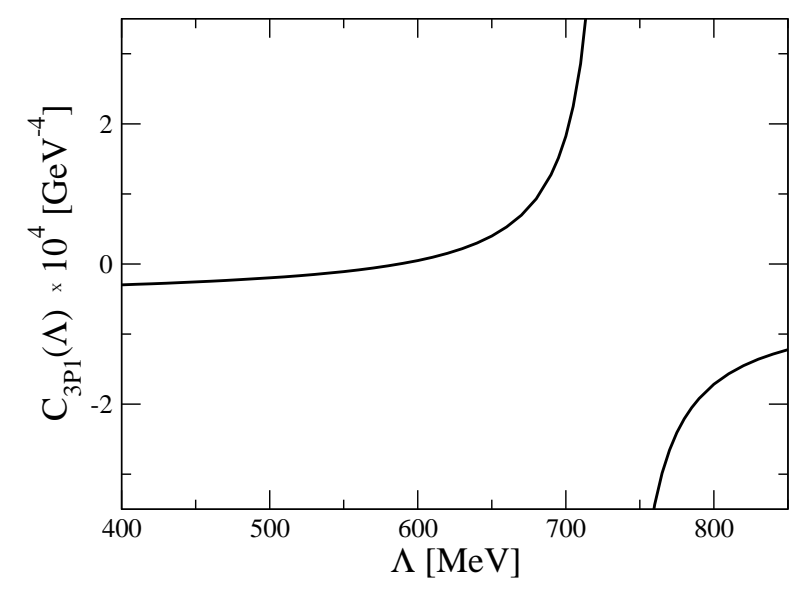

Fig. 6. "Running" of the LEC $C_{3 P 1}$ with the cut-off $\Lambda$ at NNLO. The cut-off $\tilde{\Lambda}$ in the spectral function representation is fixed at our central value $\tilde{\Lambda}=600 \mathrm{MeV}$.

obtained from fit to NN phase shifts in the EFT approach and the ones resulting from the OBE models.

\section{Summary and conclusions}

In this paper we have considered the two-nucleon potential in chiral effective field theory, making use of the novel method of regularizing the pion loop integrals introduced in I. For the low partial waves and the deuteron considered here, one has to solve the LS equation with the properly regulated potential, cf. Eq. (2.13). One thus has to deal with two different cut-offs, the first one related to the spectral function regularization (denoted $\tilde{\Lambda}$ ) and the second one related to the regularization of the potential in the LS equation (denoted $\Lambda$ ). As pointed out, both of these cut-offs can be chosen in the same range. We obtain the following results:

1) We have varied the cut-off $\Lambda$ in the LS equation in the range $450 \ldots 600 \mathrm{MeV}$ at NLO and $450 \ldots 650 \mathrm{MeV}$ at NNLO, which is a significantly larger range than in Ref. 11. The cut-off $\tilde{\Lambda}$ is varied independently in the range $500 \ldots 700 \mathrm{MeV}$, which is completely consistent with the variation of $\Lambda$.

2) As shown in Fig. 1 we obtain a modest/satisfactory description of the S-waves at NLO/NNLO when fitting the Nijmegen PSA for energies below $100 \mathrm{MeV}$. Note that the NNLO result only differs from the NLO one by inclusion of subleading two-pion exchange corrections. The LECs $c_{1,3,4}$ which enter this subleading TPE contributions are consistent with the analysis of pionnucleon scattering in chiral perturbation theory. The resulting $\mathrm{S}$-wave scattering lengths, effective ranges and higher order range parameters come out in good agreement with the ones deduced from the Nijmegen PSA, cf. Tables 1 and 2] In contrast to earlier work 8 employing dimensional regularization, we have no unphysical deeply bound states in the $\mathrm{S}$-waves (and any other partial wave).
3) At NNLO, all $\mathrm{P}$-waves and the mixing parameter $\epsilon_{1}$ are well described at NNLO below $E_{\text {lab }} \sim 100 \mathrm{MeV}$ and improved as compared to their NLO representations (one parameter per phase at NLO and NNLO), with the exception of the ${ }^{3} P_{0}$ partial wave. We stress that at NNNLO this deficiency is cured [5].

4) The D- and higher partial waves come out similar to the results obtained in Born approximation in I. For further discussion, we refer to that paper.

5) The deuteron properties collected in Table 3 are given parameter-free. We find a slight improved in the quadrupole moment as compared to the calculation based on dimensional regularization (as long as the binding energy is not used as an input parameter).

6) The theoretical error bars on the various partial waves and the bound state properties come out consistently with expectations, see in particular the discussion in the beginning of Section 3 .

7) We have shown that the numerical values of the fournucleon LECs are in good agreement with the ones derived from semi-phenomenological boson exchange models once the two-pion-exchange contribution is properly accounted for, as detailed in the appendix. This strengthens the conclusions of Ref. [12] and bridges the gap between the chiral EFT approaches and more phenomenological models describing the forces between two nucleons.

It is now of utmost importance to investigate the next order, that is the NNNLO corrections, to a achieve a truly accurate description of all important partial waves. Work along these line is underway [5] (for a first attempt using dimensional regularization see [22]).

\section{Acknowledgments}

This work is supported in part by the Deutsche Forschungsgemeinschaft (E.E.).

\section{A Reduction of the two-pion exchange contributions}

As stated before, we have to add the contribution of the TPE to the LECs so as to be able to compare with boson exchange potentials. Expanding the explicit expressions for the renormalized TPE potential given before in powers of $\mathbf{q}$ allows for a mapping on the spectroscopic LECs (of course, the TPE contains many other contributions, which are, however, of no relevance for this discussion). At NLO we get

$$
\begin{aligned}
& \tilde{C}_{1 S 0}^{\mathrm{NLO}}=-\frac{1}{3} \tilde{C}_{3 S 1}^{\mathrm{NLO}}=18 \alpha\left(1+4 g_{A}^{2}-8 g_{A}^{4}\right) M_{\pi}^{2} \tilde{\Lambda}^{2}, \\
& C_{1 S 0}^{\mathrm{NLO}}=3 \alpha\left[\left(2+17 g_{A}^{2}-88 g_{A}^{4}\right) \tilde{\Lambda}^{2}\right. \\
& \left.-2\left(1+4 g_{A}^{2}-8 g_{A}^{4}\right) M_{\pi}^{2}\right], \\
& C_{3 S 1}^{\mathrm{NLO}}=9 \alpha\left[-\left(2+17 g_{A}^{2}-40 g_{A}^{4}\right) \tilde{\Lambda}^{2}\right.
\end{aligned}
$$




$$
\begin{aligned}
& \left.+2\left(1+4 g_{A}^{2}-8 g_{A}^{4}\right) M_{\pi}^{2}\right], \\
& C_{\epsilon 1}^{\mathrm{NLO}}=-54 \sqrt{2} \alpha g_{A}^{4} \tilde{\Lambda}^{2}, \\
& C_{1 P 1}^{\mathrm{NLO}}=6 \alpha\left[\left(2+17 g_{A}^{2}-16 g_{A}^{4}\right) \tilde{\Lambda}^{2}\right. \\
& \left.-2\left(1+4 g_{A}^{2}-8 g_{A}^{4}\right) M_{\pi}^{2}\right], \\
& C_{3 P 0}^{\mathrm{NLO}}=-2 \alpha\left[\left(2+17 g_{A}^{2}+74 g_{A}^{4}\right) \tilde{\Lambda}^{2}\right. \\
& \left.-2\left(1+4 g_{A}^{2}-8 g_{A}^{4}\right) M_{\pi}^{2}\right], \\
& C_{3 P 1}^{\mathrm{NLO}}=2 \alpha\left[-\left(2+17 g_{A}^{2}-61 g_{A}^{4}\right) \tilde{\Lambda}^{2}\right. \\
& \left.+2\left(1+4 g_{A}^{2}-8 g_{A}^{4}\right) M_{\pi}^{2}\right], \\
& C_{3 P 2}^{\mathrm{NLO}}=2 \alpha\left[-\left(2+17 g_{A}^{2}-7 g_{A}^{4}\right) \tilde{\Lambda}^{2}\right. \\
& \left.+2\left(1+4 g_{A}^{2}-8 g_{A}^{4}\right) M_{\pi}^{2}\right],
\end{aligned}
$$

Finally, it should be kept in mind that we use here the same notation as in our previous work [8], according to which the contact terms that are nonanalytic in the pion mass and result from TPE, see e.g. I, are not shown explicitly. In practical applications to the NN system at fixed value of $M_{\pi}$ such terms cannot be disentangled from the zero-range counter terms (contact interactions from the Lagrangian) and therefore do not need to be treated separately. A more precise treatment is required if the pion mass dependence of the nucleon force is studied 23. 24,25.

\section{References}

1. E. Epelbaum, W. Glöckle, and U.-G. Meißner, nucl-th/0304037 Eur. Phys. J. A (2003) in press.

2. N. Kaiser, R. Brockmann, and W. Weise, Nucl. Phys. A 625 (1997) 758.

where

$$
\alpha=\frac{\sqrt{\tilde{\Lambda}^{2}-4 M_{\pi}}}{432 F_{\pi}^{4} \tilde{\Lambda}^{3} \pi} .
$$

The above expressions coincide exactly with the ones given in Ref. 12 in the limit $\tilde{\Lambda} \rightarrow \infty$. Similarly, we can give the additional TPE NNLO contributions to the various LECs:

$$
\begin{aligned}
& \tilde{C}_{1 S 0}^{\mathrm{NNLO}}=\tilde{C}_{3 S 1}^{\mathrm{NNLO}}=-36 \beta\left(2 c_{1}-c_{3}\right) M_{\pi}^{2} \tilde{\Lambda}^{2}, \\
& C_{1 S 0}^{\mathrm{NNLO}}=C_{3 S 1}^{\mathrm{NNLO}}=3 \beta\left[-4 c_{4} \tilde{\Lambda}^{2}\right. \\
& -2 c_{1}\left(5 \tilde{\Lambda}^{2}-2 \tilde{\Lambda} M_{\pi}-4 M_{\pi}^{2}\right) \\
& \left.+c_{3}\left(11 \tilde{\Lambda}^{2}-2 \tilde{\Lambda} M_{\pi}-4 M_{\pi}^{2}\right)\right] \text {, } \\
& C_{\epsilon 1}^{\mathrm{NNLO}}=12 \sqrt{2} \beta c_{4} \tilde{\Lambda}^{2}, \\
& C_{1 P 1}^{\mathrm{NNLO}}=2 \beta\left[-12 c_{4} \tilde{\Lambda}^{2}+2 c_{1}\left(5 \tilde{\Lambda}^{2}-2 \tilde{\Lambda} M_{\pi}-4 M_{\pi}^{2}\right)\right. \\
& \left.-c_{3}\left(11 \tilde{\Lambda}^{2}-2 \tilde{\Lambda} M_{\pi}-4 M_{\pi}^{2}\right)\right], \\
& C_{3 P 0}^{\mathrm{NNLO}}=2 \beta\left[-8 c_{4} \tilde{\Lambda}^{2}+2 c_{1}\left(5 \tilde{\Lambda}^{2}-2 \tilde{\Lambda} M_{\pi}-4 M_{\pi}^{2}\right)\right. \\
& \left.-c_{3}\left(11 \tilde{\Lambda}^{2}-2 \tilde{\Lambda} M_{\pi}-4 M_{\pi}^{2}\right)\right], \\
& C_{3 P 1}^{\mathrm{NNLO}}=2 \beta\left[2 c_{4} \tilde{\Lambda}^{2}+2 c_{1}\left(5 \tilde{\Lambda}^{2}-2 \tilde{\Lambda} M_{\pi}-4 M_{\pi}^{2}\right)\right. \\
& \left.-c_{3}\left(11 \tilde{\Lambda}^{2}-2 \tilde{\Lambda} M_{\pi}-4 M_{\pi}^{2}\right)\right], \\
& C_{3 P 2}^{\mathrm{NNLO}}=2 \beta\left[-2 c_{4} \tilde{\Lambda}^{2}+2 c_{1}\left(5 \tilde{\Lambda}^{2}-2 \tilde{\Lambda} M_{\pi}-4 M_{\pi}^{2}\right)\right. \\
& \left.-c_{3}\left(11 \tilde{\Lambda}^{2}-2 \tilde{\Lambda} M_{\pi}-4 M_{\pi}^{2}\right)\right],
\end{aligned}
$$

where

$$
\beta=\frac{g_{A}^{2} M_{\pi}\left(\tilde{\Lambda}-2 M_{\pi}\right)}{48 F_{\pi}^{4} \tilde{\Lambda}^{3}} .
$$

These expressions depend on the dimension two LECs $c_{1,3,4}$ as discussed before and coincide with the ones given in Ref. [12] in the limit $\tilde{\Lambda} \rightarrow \infty$ modulo $1 / m$-corrections, which are not considered in the present work.
3. P. Büttiker, and U.-G. Meißner, Nucl. Phys. A 668 (2000) 97.

4. D.R. Entem, and R. Machleidt, Phys. Rev. C 66 (2002) 014002.

5. E. Epelbaum, U.-G. Meißner, and W. Glöckle, in preparation.

6. V. Bernard, Th.R. Hemmert, and U.-G. Meißner, hep-ph/0307115

7. M.C.M. Rentmeester, R.G.E. Timmermans, and J.J. de Swart, Phys. Rev. C67 (2003) 044001.

8. E. Epelbaum, W. Glöckle, and U.-G. Meißner, Nucl. Phys. A671 (2000) 295.

9. P. Lepage, nucl-th/9706029

10. J. Gegelia and G. Japaridze, Phys. Lett. B517 (2001) 476.

11. E. Epelbaum, A. Nogga, W. Glöckle, H. Kamada, U.-G.

12. E. Epelbaum, U.-G. Meißner, W. Glöckle, and Ch. Elster, Phys. Rev. C65 (2002) 044001.

13. V.G.J. Stoks, R.A.M. Klomp, M.C.M. Rentmeester, and J.J. de Swart, Phys. Rev. C48 (1993) 792.

14. T.D. Cohen and J.M. Hansen, Phys. Rev. C59 (1999) 13.

15. M.C.M. Rentmeester, private communication, 1999.

16. J.J. de Swart, C.P.F. Terheggen, and V.G.J. Stoks, nucl-th/9509032

17. D. Phillips, AIP Conf.Proc. 603 (2001) 149.

18. M. Walzl and U.-G. Meißner, Phys. Lett. B513 (2001) 37.

19. R. Machleidt, Adv. Nucl. Phys. 19 (1989) 189.

20. V.G.J. Stoks et al., Phys. Rev. C49 (1994) 2950.

21. P.F. Bedaque, H.-W. Hammer, and U. van Kolck, Phys. Rev. Lett. 82 (1999) 463.

22. D. R. Entem and R. Machleidt, arXiv:nucl-th/0304018

23. E. Epelbaum, U.-G. Meißner, and W. Glöckle, Nucl. Phys. A714 (2003) 535.

24. S. R. Beane and M. J. Savage, Nucl. Phys. A713 (2003) 148.

25. S. R. Beane and M. J. Savage, Nucl. Phys. A717 (2003) 91. Meißner and H. Witala, Eur. Phys. J. A15 (2002) 543. 